\title{
Gastric mucosal inflammation and epithelial cell turnover are associated with gastric cancer in patients with Helicobacter pylori infection
}

\author{
T Yoshimura, T Shimoyama, M Tanaka, Y Sasaki, S Fukuda, A Munakata
}

\begin{abstract}
Background-Infection with a virulent Helicobacter pylori strain is associated with gastric mucosal damage and the increased risk of gastric cancer.

Aims-To examine the characteristics of host gastric mucosal responses in patients with gastric cancer, histological grade of gastritis, gastric epithelial apoptosis, and proliferation were studied.

Methods-Thirty two patients with early gastric cancer and 32 sex and age matched controls were studied. All subjects were infected with a virulent $H$ pylori strain (vacA $\mathrm{s} 1 / \mathrm{m} 1$, cagA positive genotype). Biopsy specimens were taken from the antrum and the corpus of the stomach. The grade of gastritis was assessed according to the updated Sydney system. Apoptotic cells were detected using terminal uridine deoxynucleotidyl nick end labelling, and epithelial cell proliferation was determined by means of the Ki-67 labelling index.

Results-In patients with gastric cancer, significantly higher grades were observed when glandular atrophy $(p<0.05)$ and intestinal metaplasia $(p<0.01)$ were present in the antrum, and when mononuclear cell infiltration was present in the corpus $(p<0.05)$. The numbers of apoptotic cells were increased in patients with cancer $(p<0.05)$ and the apoptotic index correlated significantly with the grade of glandular atrophy. Epithelial cell proliferation was more likely to be increased in mucosa where intestinal metaplasia was present.
\end{abstract}

First Department of Internal Medicine, Hirosaki University School of Medicine, 5 Zaifu-cho, Hirosaki 036-8562, Japan

$\mathrm{T}$ Yoshimura

T Shimoyama

Y Sasaki

S Fukuda

A Munakata

Department of Pathology, Hirosaki

University School of

Medicine

M Tanaka

Correspondence to:

Dr Shimoyama

email:

tsimo-hki@umin.u-tokyo.ac.jp

Accepted for publication

7 January 2000 $(\text { IARC) })^{1}$ and the World Health Organisation (WHO). ${ }^{2}$ Many studies have noted an association between particular strains of $H$ pylori and the development of gastric cancer. In Western countries, infection with a virulent $H$ pylori strain is recognised to be associated with the development of gastric cancer. Infection with an $H$ pylori strain possessing the cytotoxin associated gene A (cagA) causes enhanced gastric epithelial proliferation and apoptosis. ${ }^{3}$ Recent serological studies have shown that CagA seropositivity is associated with an increased risk of atrophic gastritis and gastric cancer. ${ }^{5}$ Vacuolating cytotoxin (VacA), encoded by the vacA gene, induces cytoplasmic vacuolation in eukaryotic cells. ${ }^{6}$ VacA production has been associated with the development of atrophic gastritis. ${ }^{7}$ Divergency in the vacA gene has been also examined and $H$ pylori strains with the vacA $\mathrm{s} 1 \mathrm{a} / \mathrm{m} 1$ genotype are associated with enhanced gastric inflammation. ${ }^{89}$ However, in Japan, where most $H$ pylori are of the vacA $\mathrm{s} 1 \mathrm{a} / \mathrm{m} 1$, cagA positive genotype, strain diversity has not been associated with gastric cancer. ${ }^{10}{ }^{11}$ Therefore, other factors might contribute to the development of gastric cancer in patients infected with H pylori.

Recent interest has been focused on the host responses to infection with $H$ pylori. Chronic gastritis induced by $H$ pylori infection usually progresses to atrophic gastritis, which is a well known risk factor for gastric cancer. Gastric mucosal integrity is maintained by cell loss (apoptosis) balanced with cell proliferation. Because severe atrophic gastritis is usually seen in the background mucosa of patients with gastric cancer, the balance of gastric epithelial cell turnover is possibly altered. Although increased gastric epithelial apoptosis and/or cell proliferation are induced by infection with $H$ pylori, ${ }^{12-15}$ different strains have been shown to have varying degrees of effect on gastric epithelial apoptosis and proliferation. ${ }^{3}$ Consequently, to study differences in host responses, $H$ pylori should be of the same genotype. However, no study has compared epithelial cell turnover in patients with gastric cancer with that seen in patients with chronic gastritis by means of a case control study where all patients are infected with the same $H$ pylori genotype.

To compare gastric mucosal responses to infection with $H$ pylori between patients with gastric cancer and chronic gastritis, we examined the grade of gastritis and gastric epithelial cell turnover in a case control study matching the cagA and vacA genotype of the infected strain. We also examined the relation of gastric epithelial cell proliferation and apoptosis to the histological grades of gastritis to understand 
the mechanism of development of atrophic gastritis and gastric cancer.

\section{Materials and methods}

PATIENTS

Patients with cancer and control subjects were selected from patients who were scheduled for upper gastrointestinal endoscopy for routine screening for gastric cancer at Hirosaki University Hospital. We excluded patients who had received anti-ulcer agents or antibiotics for up to two months before the examination and those who had histories of gastric cancer, gastric or duodenal ulcer, or gastric surgery. All patients provided informed consent before endoscopy. Patients with cancer were enrolled into our study when their diagnosis was histologically confirmed. Control subjects were eligible if their endoscopic diagnosis was normal, or if atrophic gastritis was present without any evidence of ulceration. Gastric biopsy specimens were taken for $H$ pylori culture, histological analysis, and studies of epithelial cell turnover. In patients with gastric cancer, biopsy specimens were taken at least $2 \mathrm{~cm}$ away from the tumours. Thirty two patients with early gastric cancer (age range, 43-74 years; mean age, 60; 21 men and 11 women) and 32 sex and age matched control subjects were enrolled into our study.

Biopsy specimens were cultured for three to five days on Skirrow blood agar at $37^{\circ} \mathrm{C}$. The selected clones were suspended in $1 \mathrm{ml}$ phosphate buffered saline (PBS; pH 7.6) for DNA extraction. The presence of the cagA gene and the allelic variation of the vacA gene was determined by the polymerase chain reaction (PCR) using previously described primers. ${ }^{16} \mathrm{~Pa}-$ tients with cancer and control subjects were eligible if they were infected with a cagA positive, vacA s1/m1 strain. ${ }^{10} 11$ Our study was approved by the ethics committee of Hirosaki University.

\section{HISTOLOGICAL ANALYSIS}

Biopsy specimens from the antrum and the corpus of the stomach were embedded in paraffin wax, and stained with haematoxylin-eosin and with the Warthin-Starry method. Mononuclear cell infiltration, polymorphonuclear cell infiltration, glandular atrophy, intestinal metaplasia, and the density of $H$ pylori were graded from 0 to 3 , according to the updated Sydney system, ${ }^{18}$ by an experienced pathologist. The intraobserver reproducibility was assessed on 30 antral biopsy specimens used in our study. The calculated $\kappa$ value was 0.81 for the grade of glandular atrophy and similar values were seen for other histological features. A full histological diagnosis of the tumour type and stage was undertaken on resected specimens, and the gastric cancer was classified into intestinal type or diffuse type according to the Lauren system. ${ }^{19}$ Histologically, 26 patients had intestinal type gastric cancer and six patients had diffuse type gastric cancer.

DETERMINATION OF APOPTOSIS

A characteristic of apoptosis is the stepwise degradation of DNA by endonucleases, result- ing in many short fragments of double stranded DNA. Apoptotic cells can be identified by incorporating labelled nucleotides into the $3^{\prime}$ free hydroxyl ends using terminal deoxynucleotidyl transferase and visualising them histochemically, by means of terminal uridine deoxynucleotide nick end labelling (TUNEL).$^{20}$ According to this principle, apoptotic cells were stained with the apoptosis detection kit (Oncor, Gaithersburg, USA). Paraffin wax embedded gastric mucosal specimens were cut at $4 \mu \mathrm{m}$ and mounted on to microscope slides. The specimens were washed with xylene and ethanol to remove paraffin wax, rinsed with Tris buffer $(\mathrm{pH} \mathrm{7.4)}$, and digested for 15 minutes at room temperature with $40 \mu \mathrm{g} / \mathrm{ml}$ proteinase K (Sigma, Poole, Dorset, UK). After washing with Tris buffer and blocking of endogenous peroxidase with $3 \% \mathrm{H}_{2} \mathrm{O}_{2}$ for 30 minutes, sections were incubated with digoxigenin-dUTP at $37^{\circ} \mathrm{C}$ for one hour. Detection of incorporated digoxigenin was carried out with antidigoxigeninperoxidase conjugated antibody. The sections were washed with buffered saline, stained in $0.05 \%$ diaminobenzidine hydrochloride (DAB) solution for five minutes, and counterstained in haematoxylin for five seconds. After dehydration with xylene, the sections were mounted under a glass coverslip. The numbers of positive cells in 10 whole glands were counted at three levels from the same biopsy of each patient, and this was considered to be the apoptotic index.

\section{DETERMINATION OF CELL PROLIFERATION}

$\mathrm{Ki}-67$ is a nuclear antigen that is expressed at all stages of the cell cycle, apart from G0, and the anti-Ki-67 antibody, MIB-1, is useful for the analysis of cell proliferation in paraffin wax embedded sections. ${ }^{21}$ Cell proliferation in gastric epithelium was examined by the $\mathrm{Ki}-67$ labelling index. The specimens were dewaxed and microwaved (Bio-Rad H2500) in $0.01 \mathrm{M}$ citrate buffer for 20 minutes. The MIB- 1 antibody was applied and the slides were incubated at $4^{\circ} \mathrm{C}$ for 14 hours. The specimens were stained using a streptavidin-biotin technique. The number of positive cells/100 gastric mucosal epithelial cells was counted in three sections (finally 300 cells were counted) and the mean number was considered to be the proliferation index.

\section{STATISTICAL ANALYSIS}

The Mann-Whitney $U$ test was used to compare the grade of histological features of gastritis. The two tailed $t$ test was performed to examine the difference in the apoptotic index and the cell proliferation index between cancer patients and control subjects. Spearman's correlation coefficient was calculated to examine the correlation between the grade of gastritis and gastric epithelial cell turnover. A $p$ value of less than 0.05 was considered significant.

\section{Results}

HISTOLOGICAL ANALYSIS

All patients and control subjects studied were infected with a virulent $H$ pylori strain (vacA 
Table 1 The grade of histological features of gastritis in the antrum and the corpus

\begin{tabular}{|c|c|c|c|c|c|c|c|c|c|}
\hline & \multicolumn{4}{|c|}{ Cancer patients } & \multicolumn{4}{|c|}{ Control subjects } & \multirow[b]{2}{*}{ p Value } \\
\hline & 0 & 1 & 2 & 3 & 0 & 1 & 2 & 3 & \\
\hline \multicolumn{10}{|l|}{ Antrum } \\
\hline MNC infiltration & 0 & 6 & 18 & 8 & 1 & 5 & 23 & 3 & NS \\
\hline PMN infiltration & 12 & 18 & 2 & 0 & 11 & 19 & 2 & 0 & NS \\
\hline Glandular atrophy & 5 & 2 & 12 & 13 & 4 & 11 & 13 & 4 & $\mathrm{p}<0.05$ \\
\hline Helicobacter pylori density & 6 & 14 & 4 & 8 & 4 & 11 & 8 & 9 & NS \\
\hline Intestinal metaplasia & 13 & 5 & 7 & 7 & 23 & 3 & 5 & 1 & $\mathrm{p}<0.01$ \\
\hline Corpus & & & & & & & & & \\
\hline MNC infiltration & 0 & 4 & 21 & 7 & 4 & 6 & 19 & 3 & $\mathrm{p}<0.05$ \\
\hline PMN infiltration & 6 & 20 & 6 & 0 & 11 & 11 & 10 & 0 & NS \\
\hline Glandular atrophy & 11 & 12 & 4 & 5 & 17 & 4 & 6 & 5 & NS \\
\hline Helicobacter pylori density & 1 & 9 & 9 & 13 & 5 & 10 & 5 & 12 & NS \\
\hline Intestinal metaplasia & 24 & 2 & 4 & 2 & 27 & 2 & 2 & 1 & NS \\
\hline
\end{tabular}

Data represent the number of patients.

p Values were determined by Mann-Whitney U test.

MNC, mononuclear cell; NS, not significant; PMN, polymorphonuclear cell.

$\mathrm{s} 1 / \mathrm{m} 1$, cagA positive genotype). Biopsy specimens were obtained from all patients for histological analysis. Table 1 summarises the grades of histological features in the antrum and the corpus. Helicobacter pylori was present histologically in all patients. In the antral mucosa, the grade of glandular atrophy and intestinal metaplasia was significantly higher in patients with gastric cancer than in control subjects ( $\mathrm{p}<0.05$ and $\mathrm{p}<0.01$, respectively). The grades of mononuclear cell infiltration, polymorphonuclear cell infiltration, and $H$ pylori density were not significantly different. In contrast, in the corpus mucosa, mononuclear cell infiltration was significantly higher in patients with gastric cancer $(p<0.05)$. No significant differences were observed in other histological features. In patients with intestinal type gastric cancer, the grade of atrophy was significantly higher than that in control subjects $(\mathrm{p}<0.01)$. Intestinal metaplasia was also significantly more severe in patients with intestinal type gastric cancer than in control subjects in both the antrum $(\mathrm{p}<0.01)$ and the corpus $(\mathrm{p}<0.05)$. Intestinal metaplasia was seen in 19 patients with intestinal type gastric cancer; 10 were of the incomplete type and nine were of the complete type. In two patients with diffuse type gastric cancer, one had incomplete and another had complete type metaplasia. Intestinal metaplasia was also seen in 10 control subjects; four were incomplete and six were of the complete type. The proportion of incomplete and complete intestinal metaplasia was similar between the groups.

EPITHELIAL CELL PROLIFERATION AND APOPTOSIS Sections for the calculation of the apoptotic index were available in 16 patients with intestinal type gastric cancer, four patients with diffuse type gastric cancer, and their matched controls. In the antral mucosa, the mean (SEM) apoptotic index was $1.04(0.33)$ in patients with intestinal type gastric cancer, $1.42(0.77)$ in patients with diffuse type gastric cancer, and $0.62(0.19)$ in control subjects. In the corpus mucosa, it was $0.65(0.21)$ in patients with intestinal type gastric cancer, $0.41(0.32)$ in diffuse type gastric cancer, and $0.20(0.07)$ in control subjects. The mean apoptotic index was higher in patients with intestinal type gastric cancer compared with

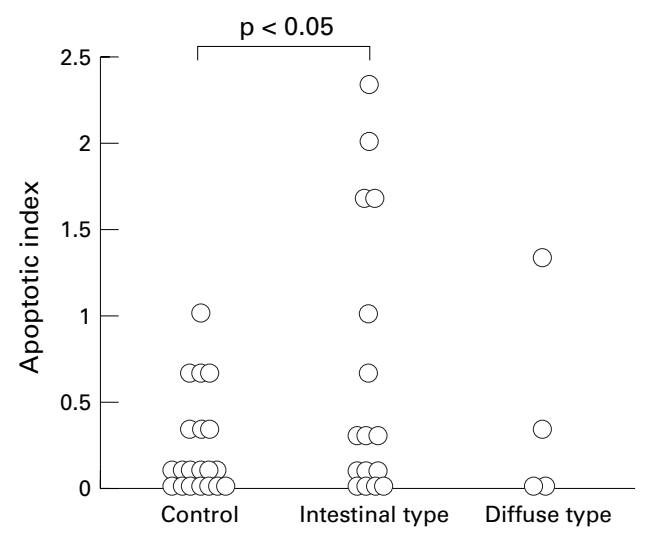

Figure 1 Apoptotic index in the corpus of the stomach in patients with intestinal and diffuse type gastric cancer and control subjects.

that seen in control subjects in the corpus ( $\mathrm{p}<0.05$; fig 1). The TUNEL labelled apoptotic bodies were seen only in gastric epithelial cells and not in metaplastic cells. The mean cell proliferation index was highest in the antrum of patients with gastric cancer when increased intestinal metaplasia was present. However, the cell proliferation index was not significantly different between the groups. In patients with intestinal type gastric cancer, the mean (SEM) cell proliferation index was $6.98(2.36)$ in the antrum and $8.60(2.37)$ in the corpus. In patients with diffuse type gastric cancer, the mean (SEM) cell proliferation index in the antrum and the corpus were 4.58 (2.16) and 6.93 (4.09), respectively. In control subjects, these values were $6.50(1.93)$ in the antrum and $5.46(1.55)$ in the corpus.

\section{CORRELATION OF GASTRITIS SCORE WITH} PROLIFERATION AND APOPTOSIS

The associations between grade of gastritis, proliferation, and apoptosis were examined. Because the apoptotic index was zero in the corpus mucosa of most control subjects and the number of patients with diffuse type cancer was too small, no correlation was found. In the antrum of control subjects, the apoptotic index correlated significantly with the grade of glandular atrophy (fig 2). However, correlations between grade of gastritis and either cell proliferation or apoptosis were not significant.

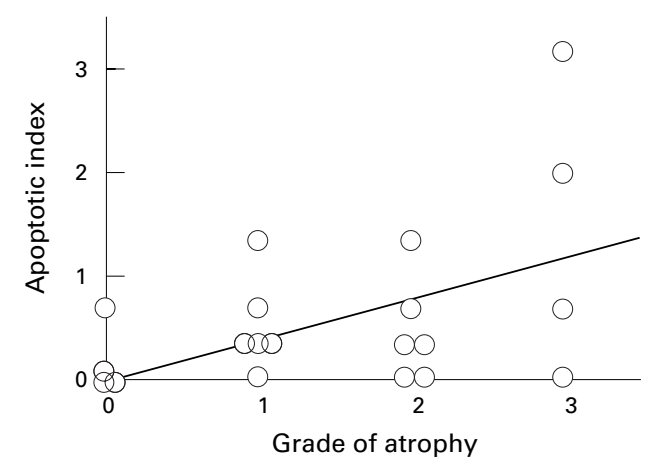

Figure 2 Correlation between apoptotic index and the grade of glandular atrophy in the corpus mucosa in patients without cancer $(r=0.467 ; 95 \%$ confidence interval, 0.031 to $0.754 ; p=0.037)$. The line represents the regression line. 


\section{Discussion}

In our study, we examined the differences in gastric mucosal responses to $H$ pylori infection by means of a case control study where all patients were infected with the same genotype. The correlations between the grade of gastritis, gastric epithelial cell proliferation, and apoptosis were also studied.

Many studies have examined the risk factors for gastric cancer. Among them, atrophic gastritis and intestinal metaplasia are well recognised to increase the risk of gastric cancer. The risk increases with the degree and the extent of atrophic gastritis. ${ }^{22-24}$ In our study, although the $H$ pylori density was not significantly different between patients with cancer and control subjects, glandular atrophy and intestinal metaplasia were significantly more severe in patients with gastric cancer than in controls. Mononuclear cell infiltration was also significantly higher in patients with gastric cancer in the corpus, whereas there was no significant difference in the antrum. These results might be a consequence of the extent of atrophic gastritis and intestinal metaplasia in the antrum. Atrophic gastritis is considered to be a result of chronic active gastritis; once severe glandular atrophy has developed, intestinal metaplasia occurs, accompanied by a reduction of $H$ pylor $i$ density. This results in a reduction in inflammatory cell infiltration. In general, more pronounced inflammation is induced by $H$ pylori in the antrum than in the corpus. ${ }^{25}$ In our study, however, severe glandular atrophy and intestinal metaplasia might have contributed to the reduced inflammatory infiltrate in the antrum of patients with gastric cancer.

Increased apoptosis and/or insufficient cell proliferation of the gastric epithelium might cause glandular atrophy. Previous studies have reported that infection with $H$ pylori increased the number of apoptotic cells in the gastric mucosa. ${ }^{12} 13$ In our study, an increased apoptotic index was found in patients with gastric cancer. Gland crypt length might vary with the degree of glandular atrophy. Because a higher apoptotic index was seen in patients with gastric cancer, despite the fact that glandular atrophy was more severe, the apoptotic index might be greater if the index was calculated on the basis of the number of epithelial cells. In contrast, gastric epithelial cell proliferation was not significantly different between patients with gastric cancer and controls. These findings suggest that epithelial cell proliferation could be insufficient to balance the increased cell loss in patients with gastric cancer. Furthermore, there was a positive correlation between the apoptotic index and the grade of glandular atrophy. Thus, the increase of apoptotic cells might play an important role in the development of glandular atrophy. ${ }^{26}$ Several studies have reported that VacA impairs gastric epithelial cell migration and proliferation..$^{27} 28$ Because all patients with cancer and controls in this study were infected with an $H$ pylori strain associated with the production of VacA, epithelial cell proliferation was possibly inhibited both in patients with gastric cancer and in control subjects. This mechanism might play a role in the development of atrophic gastritis observed in control subjects.

In our study, a higher proliferation index was seen in gastric mucosa where intestinal metaplasia was observed, although the difference was not significant. Because proliferation has been shown to be greater in gastric mucosa with intestinal metaplasia than in mucosa with gastritis only,,$^{290}$ the presence of intestinal metaplasia might contribute to the differences in epithelial cell proliferation seen in our study. Proliferating cells are known to be sensitive to mutagenic factors. The results were consistent with the fact that gastric cancer occurs frequently in mucosa with intestinal metaplasia.

Gastric cancer has been associated with infection with $\mathrm{H}$ pylori. ${ }^{12}$ Our results suggest possible roles for $H$ pylori infection in the development of gastric cancer. The presence of atrophic gastritis increases the risk of gastric cancer, which increases with the degree and the extension of atrophy. ${ }^{24}$ In our study, infection with $H$ pylori induced more severe glandular atrophy and subsequent intestinal metaplasia in patients with gastric cancer. Helicobacter pylori infection could act as a promoter for gastric cancer, through the development of atrophic gastritis and intestinal metaplasia. On the other hand, increased DNA damage in gastric epithelium induced by $H$ pylori has been associated with the development of gastric cancer. $^{31}{ }^{32}$ Apoptosis detected by the TUNEL technique shows DNA breaks in nuclei, which results from the elimination of severely damaged DNA. ${ }^{20}$ Our data showed more apoptotic epithelial cells in patients with gastric cancer than in control subjects, suggesting that increased DNA damage might occur in the gastric epithelium of patients with gastric cancer. Therefore, $H$ pylori infection might also be an initiator of gastric cancer, by inducing gastric epithelial DNA damage.

Our study was retrospective and thus we could not preclude the possibility that patients with cancer might have longer standing gastritis than control subjects. However, the results suggest several factors that might be involved in gastric carcinogenesis in patients infected with virulent $H$ pylori strains. Infection with $H$ pylori induces more severe gastric mucosal inflammation, resulting in enhanced DNA damage in patients with gastric cancer. In response to DNA damage, apoptosis of gastric epithelial cells occurs with insufficient cell proliferation, which might be inhibited by the presence of VacA. It follows that atrophic gastritis is more extensive in patients with gastric cancer than in subjects with chronic gastritis. Differences in the extent of mucosal inflammation, which might be associated with increased gastric epithelial DNA damage, and the extent of atrophic gastritis possibly contribute to the development of gastric cancer.

1 The EUROGAST Study Group. An international association between Helicobacter pylori infection and gastric cancer. Lancet 1993;341:1359-62.

2 IARC Working Group on the Evaluation of Carcinogenic Risks to Humans. Helicobacter pylori. In: Schistsomes, liver flukes and Helicobacter pylori: views and expert opinions of an flukes and Helicobacter pylori: views and expert opinions of an
IARC working group on the evaluation of carcinogenic risks to IARC working group on the evaluation of
humans. Lyon: IARC 1994:177-240. 
3 Rokkas T, Ladas S, Liatsos C, et al. Relationship of Helicobacter pylori CagA status to gastric cell proliferation and apoptosis. Dig Dis Sci 1999; $44: 487-93$.

4 Kuipers EJ, Perez-Perez GI, Meuwissen SGM, et al. Helicobacter pylori and atrophic gastritis: importance of the cagA status. F Natl Cancer Inst 1995;87:1777-80.

5 Blaser MJ, Perez-Perez GI, Kleanthous H, et al. Infection of strains possessing cagA is associated with an increased risk of developing adenocarcinoma of the stomach. Cancer Res 1995;55:2111-15.

6 Cover TL, Blaser MJ. Purification and characterization of the vacuolating toxin from Helicobacter pylori. $7 \mathrm{Biol}$ Chem 1992;267:10570-5.

7 Fox JG, Correa P, Taylor NS, et al. High prevalence and persistence of cytotoxin-positive Helicobacter pylori strains in a population with high prevalence of atrophic gastritis. Am f Gastroenterol 1992;87:1554-60.

8 Atherton JC, Cao P, Peek RM, et al. Mosaicism in vacuolating cytotoxin alleles of Helicobacter pylori: association of specific vacA types with cytotoxin production and peptic specific vacA types with cytotoxin production
ulceration. F Biol Chem 1995;270:17771-7.

9 Atherton JC, Peek RM, Tham KT, et al. Clinical and pathological importance of heterogeneity in vacA, the vacuolating cytotoxin gene of Helicobacter pylori. Gastroenterology 1997;112:92-9.

10 Shimoyama T, Yoshimura T, Mikami T, et al. Evaluation of Helicobacter pylori vacA genotype in Japanese patients with gastric cancer. F Clin Pathol 1998;51:299-301.

11 Shimoyama T, Fukuda S, Tanaka M, et al. High prevalence of the cagA-positive Helicobacter pylori strain in Japanese asymptomatic patients and gastric cancer patients. Scand $\mathcal{f}$ Gastroenterol 1997;32:465-8.

12 Moss SF, Calam J, Wang S, et al. Induction of gastric epithelial apoptosis by Helicobacter pylori. Gut 1996;38: 498-501.

13 Piotrowiski J, Plotrowski E, Skrodzka D, et al. Induction of acute gastritis and epithelial apoptosis by Helicobacter pylori lipopolysaccharide. Scand $\mathcal{f}$ Gastroenterol 1997;32: pylori lipo

14 Fan XG, Kelleher D, Xia HX, et al. Helicobacter pylori increases proliferation of gastric epithelial cells. Gut 1996 increases prot $19-22$.

15 Bechi P, Balzi M, Becciolini A, et al. Helicobacter pylori and cell proliferation of the gastric mucosa: possible implications for gastric carcinogenesis. Am f Gastroenterol 1996;91 271-6.

16 Peek RM, Miller GG, Tham KT, et al. Detection of Helicobacter pylori gene expression in human gastric mucosa. $f$ Clin Microbiol 1995;33:28-32.

17 Atherton JC, Tewlls RJ, Hawkey CJ, et al. New, internationally applicable, polymerase chain reaction-based ty ing of Helicobacter pylori vacA. Gut 1997;40(suppl 1):A1.
18 Dixon MF, Genta RM, Yardley JH, et al. Classification and grading of gastritis. The updated Sydney system. Am f Surg

19 Lauren P. The two histological main type of gastric arcinoma: diffuse and so-called intestinal type carcinom Acta Pathol Microbiol Scand 1965;64:331-49.

20 Gavrieli Y, Sherman Y, Ben-Sasson SA, et al. Identification of programmed cell death via specific labelling of nuclear DNA fragmentation. F Cell Biol 1992;119:493-501.

21 Cattoretti G, Becker MHG, Key G, et al. Monoclonal antibodies against recombinant parts of the Ki 67 antigen MIB 1 and MIB 3 detect proliferating cells in microwaveprocessed formalin-fixed paraffin sections. F Pathol 1992; 168:357-63.

22 Wei-cheng Y, William JB, Ji-you L, et al. Precancerous gastric lesions in a population at high risk of stomach cancer. Cancer Res 1993;53:1317-21

23 Kato I, Tominaga S, Ito Y, et al. A prospective study of atrophic gastritis and cancer risk. Fpn $\mathcal{F}$ Cancer Res 1992;83:1137-42.

24 Inoue N, Tajima K, Kobayashi S, et al. Protective factor against progression from atrophic gastritis to gastric cancer-data from a cohort study in Japan. Int $\mathcal{f}$ Cancer

25 Bayerdorffer E, Lehn N, Hatz R, et al. Difference in expression of Helicobacter pylori gastritis in antrum and body. Gastroenterology 1992;102:1575-82.

26 Ricci V, Ciacci C, Zarrilli R, et al. Effect of Helicobacter pylori on gastric epithelial cell migration and proliferation in vitro: role of VacA and CagA. Infect Immun 1996;64: 2829-33.

27 Lynch DAF, Mapstone NP, Clarke P, et al. Correlation between epithelial cell proliferation and histological grading in gastric mucosa 7 Clin Pathol 1999:52:367-71.

28 Romano M, Ricci V, Popolp DA, et al. Helicobacter pylor upregulates expression of epidermal growth factor-related peptides, but inhibits their proliferative effect in MKN 28 peptides, but inhibits their proliferative effect in MKN

29 Yabuki Y, Sasano H, Tobita M, et al. Analysis of cell damage and proliferation in Helicobacter pylori-infected human gastric mucosa from patients with gastric adenocarcinoma. Am F Pathol 1997;151:821-9.

30 Lynch DA, Mapstone NP, Clarke AM, et al. Cell proliferation in Helicobacter pylori associated gastritis an the effect of eradication therapy. Gut 1995;36:346-50

31 Balik CS, Youn SH, Chung HM, et al. Increased oxidative DNA damage in Helicobacter pylori-infected human gastric mucosa. Cancer Res 1996;15:1279-82.

32 Mannuck EE, Bravo EL, Zarama G, et al. Inducible nitric oxide synthase, nitrotyrosine, and apoptosis in Helicobacter pylori gastritis: effect of antibiotics and antioxidants. Cancer Res 1996;15:3238-43. 